\title{
Random Error Analysis of Inertial Sensors output Based on Allan Variance
}

\author{
Shaochen $\mathrm{Li}^{1, \text { a }}$, Xiaojing $\mathrm{Du}^{2, \mathrm{~b}}$ and Junyi Zhai ${ }^{3, \mathrm{c}}$ \\ School of Aerospace Engineering. Beijing Institute of Technology. Beijing China \\ 981939162@qq.com
}

\begin{abstract}
Keywords: MEMS sensors; Allan variance; Mathematical simulations; actual test
Abstract:With characteristics of small volume, low cost and high reliability, MEMS inertial sensors are developing into one of the mainstream sensors in inertial navigation system, and its output precision has a great effect on the overall system precision. In this paper, the random error of inertial sensors output are mainly discussed and identified by Allan variance. To analysis the random error in the output of MEMS sensors, which contribute to improve the system precision, Mathematical simulations and actual tests are carried out.
\end{abstract}

\section{Introduction}

With the development of micro-electronic technology, the MEMS-IMU (Micro-Electronic Mechanical Inertial Measurement Unit) has made great changes in navigation System. In particularly, the emergence of micro-accelerometer and gyroscope enable the attitude and heading reference system to be smaller and cheaper.

To some extent, the output precision of inertial sensors determines the overall navigation system precision. Affected by production process and external environment, fractional error exists in the output of MEMS sensors, which can be roughly divided into deterministic error and random error. Deterministic error can be obtained and compensated by mathematical modeling while random error is relatively difficult to identify. In this paper, simulations and tests are presented to identify random error in the output of inertial sensors.

For the calibration of MIMU (micro inertial measurement unit) random error, quantity of researches have been done in drift testing and modeling such as establishing ARMA model, adopting neural network and using wavelet transform method. But these kinds of error models usually establish the equations whose orders are too high to do real-time estimation at low cost [1]. Allan variance analysis is a method based on time domain analysis. It is convenient to do detailed characterization and identification of error influence on system noise statistical characteristics.

\section{Random error analysis of MEMS}

The typical random error in output of MEMS inertial sensors mainly includes gauss white noise, random walk process, flicker noise and the exponentially-correlated process [2].

Gauss white noise. Gauss white noise with zero mean widely exists in the sensor output, its signal amplitudes to gauss distribution with mean zero $\left(\mu_{v}=0\right)$ and variance $\sigma_{v}^{2}$, while its power spectral density is uniform. The auto-correlation function is

$$
q_{v}(u)=\operatorname{cov}[v(t), v(t+u)]=\sigma_{v}^{2} \delta(u)
$$

In Eq. $1, \delta(u)$ is Dirac function. According to Wiener-Khinchine law, power spectral density (Power Spectral, Density, PSD) $S_{v}(f)$ has the following relationship with variance $\sigma_{v}^{2}$ :

$$
S_{v}(f)=\sigma_{v}^{2}
$$


Random Walk noise. Random walk noise $\xi_{\omega}(t)$ can be considered as a result of integration by zero mean gauss white noise $v_{\omega}(t)$ whose autocorrelation function is $q_{v_{\omega}}(\mu)=\sigma_{v_{\omega}}^{2} \delta(\mu)$. The autocorrelation function and variance are showed as follows:

$$
\begin{aligned}
& q_{\omega}(t, u)=\operatorname{cov}\left[\xi_{\omega}(t), \xi_{\omega}(t+u)\right] \\
& =\sigma_{v_{\omega}}^{2} \min (t, t+u) \\
& \operatorname{var}\left[\xi_{\omega}(t)\right]=q_{\omega}(t, 0)=\sigma_{v_{\omega}}^{2} t
\end{aligned}
$$

Random walk noise variance changes over time, so its power spectrum density function can be seen as gauss white noise filtered by $H(f)=1 / j 2 \pi f$ :

$$
\begin{aligned}
& S_{\xi_{\omega}}(f)=|H(f)|^{2} S_{v_{\omega}}(f) \\
& \text { Knowing } S_{v}(f)=\sigma_{v}^{2}, \\
& S_{\xi_{\omega}}(f)=\frac{\sigma_{v_{\omega}}^{2}}{(2 \pi)^{2}} \frac{1}{f^{2}}
\end{aligned}
$$

Flicker noise. Flicker noise $\xi_{b}(t)$ cannot be described with specific differential equations, but it can also be obtained by gauss white noise filtered by $H(f)=1 / \sqrt{j 2 \pi f}$. Assume the white noise autocorrelation function is:

$$
q_{v_{b}}(u)=\sigma_{v_{b}}^{2} \delta(u)
$$

The PSD of flicker noise is:

$$
S_{\xi_{b}}(f)=\frac{\sigma_{v_{b}}^{2}}{2 \pi} \frac{1}{f}
$$

Exponentially-correlated noise. Exponentially correlated noise $\xi_{e}(t)$ is a constant and zero mean random process, its autocorrelation function is:

$$
q_{e}(u)=\operatorname{cov}\left[\xi_{e}(t), \xi_{e}(t+u)\right]=\sigma_{v_{e}}^{2} e^{-\beta u}
$$

The process can be described in the time domain as:

$$
\xi_{e}(t)=-\beta \xi_{e}(t)+\sqrt{2 \beta} v_{e}(t)
$$

In formula(10), $v_{e}(t)$ is zero-mean Gaussian White noise with autocorrelation function of $q_{e}(u)=\sigma_{v_{e}}^{2} \delta(u)$, Same to random walk process, exponentially-correlated noise can also be described in the frequency domain with Gaussian white noise filtered by $H(f)=\sqrt{2 \beta} /(\beta+j 2 \pi f)$. The PSD of exponentially-correlated noise is:

$$
S_{\xi_{e}}(f)=\frac{2 \beta \sigma_{v_{e}}^{2}}{\beta^{2}+(2 \pi f)^{2}}
$$

\section{Allan variance analysis}

Allan variance analysis which can identify diversity error is an analytical method based on time domain. Its principle and mathematical expressions are as follows:

Assuming that $\bar{y}_{k}$ is the average value of a signal $y(t)$ within a period time of $\tau$, then $\bar{y}_{k}$ can be described as:

$$
\begin{aligned}
& \bar{y}_{k}=\frac{1}{\tau} \int_{t_{k}}^{t_{k+1}} y(t) d t \\
& t_{k+1}=t_{k}+\tau, k=1,2, \mathrm{~K}
\end{aligned}
$$

Allan variance of $y(t)$ can be described as: 


$$
\sigma_{A y}(\tau)=\lim _{M \rightarrow \infty} \frac{1}{M-1} \sum_{k=0}^{M-1} \frac{\left(\bar{y}_{k+1}-\bar{y}_{k}\right)^{2}}{2}
$$

With sampling time interval $T_{s}$, the signal $y(t)$ which lasts for a period time of $T$ can be denoted as $y(k) k=1,2, \ldots N\left(N=T / T_{s}\right)$. Then $y(k)$ can be divided into $K(K=N / n)$ clusters with $\mathrm{n}$ sequential data points. The average time for each cluster is $\tau=n T_{s}$. The average value of cluster $k+1$ can be found according to:

$$
\bar{y}_{k+1}(\tau)=\frac{1}{n} \sum_{i=1}^{n} y_{n k+i}
$$

The Allan variance estimation is:

$$
\hat{\sigma}_{A y}(\tau)^{2}=\frac{1}{2(K-1)} \sum_{k=1}^{K-1}\left(\bar{y}_{k+1}-\bar{y}_{k}\right)^{2}
$$

According to reference [2], the error between $\hat{\sigma}_{A y}(\tau)$ and $\sigma\left(\delta_{A v}\right)$ can be described as:

$$
\Delta \sigma\left(\delta_{A v}\right)=\frac{1}{\sqrt{2(N / n-1)}}
$$

Based on Eq.16, a conclusion can be drawn that the more data points and less number of points per data set, the higher the quality of Allan variance estimates.

\section{Simulation and test}

Mathematical simulation. Between Allan variance of random signal and the corresponding PSD, a conversion relationship exists, which can be expressed as follow:

$$
\sigma_{A y}^{2}(\tau)=4 \int_{0}^{\infty} S_{y}(f) \frac{\sin ^{4}(\pi f \tau)}{(\pi f \tau)^{2}} d f
$$

Substituting Eq.2, Eq.6, Eq.8, Eq.11 into Eq.17, then

Gauss white noise:

$$
\sigma_{A v}^{2}(\tau)=\frac{\sigma_{v}^{2}}{\tau}
$$

Random Walk noise:

$$
\sigma_{A \xi_{\omega}}^{2}(\tau)=\frac{\sigma_{v_{\omega}}^{2} \tau}{3}
$$

Flick noise:

$$
\sigma_{A \xi_{b}}^{2}(\tau)=\frac{2 \sigma_{v_{b}}^{2} \ln 2}{\pi}
$$

Exponentially-correlated noise:

According to reference [3], the random error of inertial sensors can be considered to be composed of gauss white noise and exponentially-correlated noise.

The Allan variance of Gauss white noise and exponentially-correlated noise superposition is

$$
\sigma_{A v \xi_{e}}^{2}(\tau)=\sigma_{A v}^{2}(\tau)+\sigma_{A \xi_{e}}^{2}(\tau)
$$



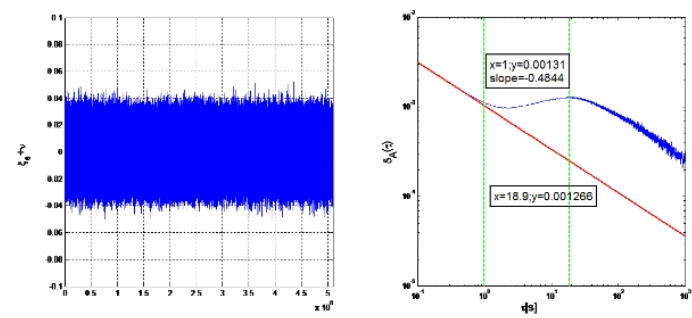

Figure 1 Gauss white noise and Exponentially-correlated noise superposition

$\sigma_{A v}^{2}(\tau)$, variance of Gaussian white noise, is inversely proportional to the time, and the variance of exponentially-correlated noise $\sigma_{A \xi_{e}}^{2}(\tau)$ increases initially and then decreases with the growth of $\tau$, reaching its maximum at $\tau=1.893 / \beta$, while $\sigma_{A v \xi_{e}}^{2}(\tau)$ also reaches its maximum at $\tau=1.893 / \beta$.

Simulation shows that $\sigma_{A v \xi_{e}}^{2}(\tau)$ reaches its maximum 0.001266 and $\sigma_{v_{e}}=0.001992$ at $\tau=18.9 \mathrm{~s}$ with $1 / \beta=10 \mathrm{~s}$ and $\sigma_{v_{e}}=0.002$. The error of $\sigma_{v_{e}}$ is within $0.8 \%$. The result shows that Allan variance can effectively distinguish the random error of inertial sensors.

Actual test. In this chapter, Allan variance is applied to analysis the actual output data of MIMU. MTI, a miniature inertial measurement unit with three-axis gyroscope and accelerometer as well as 3D magnetometers, is chosen to acquire the test data for 16 hours and a total of 5.76e6 amount.

Take the $\mathrm{x}$ axis for example, Allan variance of rate gyroscope and acceleration in $\log \sigma_{A v}(\tau)-\log \tau$ plot are showed as follows:
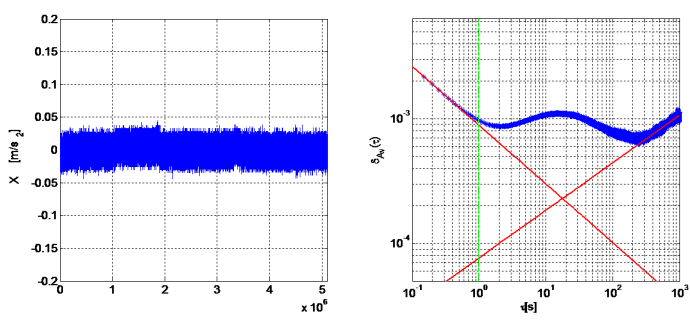

Figure 2 The $\mathrm{x}$ axis accelerometer output and Allan variance analysis
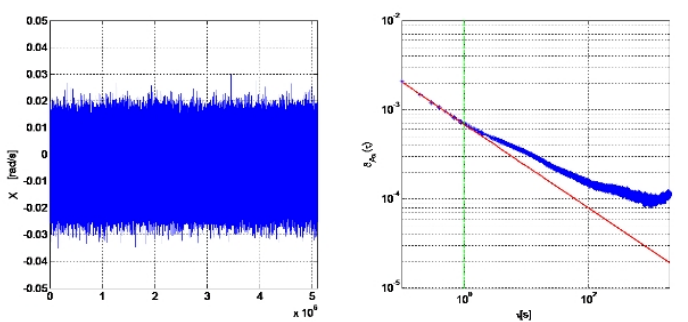

Figure 3 The $\mathrm{x}$ axis gyroscope output and Allan variance analysis

The results show that liners with -0.5 slope in $\log \sigma_{A v}(\tau)-\log \tau$ plot can be obtained when mean time $\tau$ is small, suggesting that the output of accelerometer and gyroscope contain Gaussian white noise. With Allan variance $\delta A(\tau)$ at $\tau=1, \sigma_{v}$ can be acquired.

The random error parameters of gyroscope and accelerometer are shown in Table 1 and Table 2.

Table 1 The error mode of gyroscope

\begin{tabular}{c|ccc}
\hline & \multicolumn{3}{|c}{ Gyros } \\
\hline & ARW & Gauss M arkov & $\tau$ \\
& {$[\mathrm{rad} / \sqrt{\mathrm{s}}]$} & {$[\mathrm{rad} / \mathrm{s}]$} & {$[\mathrm{s}]$} \\
\hline $\mathrm{X}$ & 0.00069 & 0.00016 & 606 \\
$\mathrm{Y}$ & 0.00058 & 0.00018 & 342 \\
$\mathrm{Z}$ & 0.00060 & 0.00017 & 313 \\
\hline
\end{tabular}


Table 2 The error mode of accelerometer

\begin{tabular}{c|ccc}
\hline & \multicolumn{3}{|c}{ Accelerometers } \\
\hline & $\begin{array}{c}\text { VRW } \\
{[m / s / \sqrt{s}]}\end{array}$ & $\begin{array}{c}\text { Gauss Markov } \\
{\left[\mathrm{m} / \mathrm{s}^{2}\right]}\end{array}$ & $\begin{array}{c}\tau \\
{[s]}\end{array}$ \\
\hline $\mathrm{X}$ & 0.00098 & 0.0018 & 35 \\
$\mathrm{Y}$ & 0.00093 & 0.0012 & 34 \\
$\mathrm{Z}$ & 0.00098 & 0.0017 & 43 \\
\hline
\end{tabular}

According to reference [4], error model of the chosen MTI is as follows:

$\omega_{i b}^{b}=\omega_{i b}^{b}+b_{\omega}+v_{\omega}, \quad f / 0=f^{b}+b_{f}+v_{f}$

$v_{\omega}, v_{f}$ are the white noises of gyroscope and accelerometer, and its intensity can be obtained by table 1. ${ }^{b_{\omega}}, b_{f}$ are the drift, including constant bias and first-order mark-off noise.

$b_{\omega}=b_{\omega o}+b_{\omega e}, b_{f}=b_{f o}+b_{f e}$

According to Eq.10, $b_{\omega e}$ and $b_{f e}$ can be expressed as:

$b_{\omega e}^{\alpha}=-\beta_{\omega} b_{\omega e}+\sqrt{2 \beta_{\omega}} v_{b \omega}$

$\delta_{f e}^{\alpha}=-\beta_{f} b_{f e}+\sqrt{2 \beta_{f}} v_{b f}$

$\beta_{\omega}, \beta_{f}$ are the mark-off process correlated time of Gyroscope and accelerometer, which can be acquired from table 1 as well as the driving noise $v_{b \omega}, v_{b f}$.

$$
\begin{aligned}
& \beta_{\omega}=\left[\begin{array}{lll}
\frac{1.89}{606} & \frac{1.89}{342} & \frac{1.89}{313}
\end{array}\right]^{T} \\
& \beta_{f}=\left[\begin{array}{lll}
\frac{1.89}{35} & \frac{1.89}{34} & \frac{1.89}{43}
\end{array}\right]^{T}
\end{aligned}
$$

\section{Conclusions}

As the output precision of sensors makes great difference in inertial system, in this paper, random error in the output of MEMS sensors is mainly identified. Based on the characteristic analysis of the random error, mathematical simulation is proceeded to obtain the typical random error curve and Allan variance-average time logarithm curve. With the attitude and heading reference system MTI, actual test is carried out to obtain the output random error parameters. The simulation and test results shows random error of inertial sensors output can be well identified with Allan variance analysis, which provides the basis for further research.

\section{Reference}

[1] Guangchun Li, He Kun Peng, jian-hui zeng, etc. The MEMS inertial device error modeling and compensation method review [J]. Journal of navigation and control, 2009, 8 (1).

[2] Karthik Naarayanan. Performance Aanlysis of Attitude Determinnation Algorithms for Low Cost Attitude Heading Reference Systems. ACM International Collegiate Programming Contest 2010.

[3] Zhao Sihao, Liu Mingquan, Feng Zhenming. MEMS inertial device error coefficient of Allan variance analysis method [J]. Chinese science, physics, mechanics, astronomy, 2010 (5) : 672-675.

[4] Xiaoyang Lan. Small spacecraft attitude determine technology research[D]. Beijing institute of technology, 2015.

[5] Kastelan D R. Design and implementation of a GPS-aided inertial navigation system for a helicopter UAV[M]. 2009. 